\title{
Joint interpolation of multi-sensor sea surface temperature fields using non-local and statistical priors
}

Ronan Fablet, Senior Member, IEEE, and François Rousseau

\begin{abstract}
This work addresses the joint analysis of multi-source and multi-resolution remote sensing data for the interpolation of high-resolution sea surface geophysical fields. As case-study application, we consider the interpolation of sea surface temperature fields. We propose a novel statistical model, which combines two key features: an exemplar-based prior and statistical priors. The exemplar-based prior, referred to as a non-local prior, exploits similarities between local patches (small field regions) to interpolate missing data areas from previously observed exemplars. This nonlocal prior also sets an explicit conditioning between the multi-sensor data. Two complementary statistical priors, namely a prior on the spatial covariance and a prior on the marginal distribution of the high-resolution details, are considered as sea surface geophysical fields are expected to depict specific spectral and marginal features in relation to the underlying turbulent ocean dynamics. We report experiments on both synthetic data and real SST data. These experiments demonstrate the contributions of the proposed combination of non-local and statistical priors to interpolate visually-consistent and geophysically-sound SST fields from multi-source satellite data. We further discuss the key features and parameterizations of this model as well as its relevance with respect to classical interpolation techniques.
\end{abstract}

\section{INTRODUCTION}

The ocean surface is monitored with a dense network of satellites. Various satellites record images of multiple ocean parameters at different resolutions. As a peculiar example, sea surface temperature (SST), which is the temperature of the thin ocean's upper layer, is a peculiar example. Microwave (MW) radiometry provides low resolution observation $\left(0.5^{\circ}-0.25^{\circ}, 25 / 50 \mathrm{~km}\right)$ while infrared (IR) sensor delivers high resolution SST measurements (up to $0.02^{\circ}, 1 \mathrm{~km}$ ). SST monitoring is of high interest as SST observations are key observations for a wide range of studies, including weather forecasting, ocean circulation, global warming impacts.

R. Fablet is with the Department of Signal and Communications at Institut Telecom/Telecom Bretagne; UMR LabSTICC ; Technopôle Brest-Iroise, Brest, France, email: ronan.fablet@telecom-bretagne.eu.

F. Rousseau is with with the Department of Image and Information Processing at Institut Telecom/Telecom Bretagne; UMR LaTIM ; Technopôle Brest-Iroise, Brest, France, email: francois.rousseau@telecom-bretagne.eu 
Beyond their intrinsic resolution, the different satellite sensors also differ in their sensitivity to the atmospheric conditions, and especially the cloud coverage. Whereas microwave radiometers involve very low missing data rates, IR sensor may result in high missing data rates (up to $90 \%$ over several consecutive days in some regions). Operational level-4 products provide interpolated fields, which typically rely on covariance-driven kriging-based approaches (e.g., [2], [28]). The joint exploitation of the multi-source multi-scale data remains however a challenge to produce geophysically-consistent interpolated fields.

In this context, we address here the interpolation of missing data in high-resolution geophysical fields under the assumption that a simultaneous low-resolution observation is available. Such multi-scale interpolation is expected to depict geophysically-consistent features, with a view to conforming to the underlying turbulent dynamics, especially: i) consistent high-resolution textured patterns, ii) non-Gaussian marginals and iii) specific spectral signatures. We propose a novel model to address this multi-scale interpolation jointly accounting for the above-mentioned constraints. The key idea is to exploit a non-local patch-based framework [14], [32], [35]. Such exemplar-based representation provides an implicit texture model, which directly exploits available observations to interpolate missing data areas.

\section{PRoblem Statement AND RELATED WORKS}

The state-of-the-art techniques for missing data interpolation in earth remote sensing mainly involve two main categories of approaches. The most popular techniques, by far, involve covariance-based geostatistical filtering. This category includes kriging methods as well as optimal interpolation schemes. They are widely used both in land remote sensing [30], [36] and ocean remote sensing [30], [33], including applications in most operational products [2], [28]. These approaches rely on the definition and/or estimation of the spatial covariance of the field to be interpolated. The interpolation is stated as the computation for each grid point of the conditional mean of the field given the observed grid points and the considered covariance model. In general, this geostatistical interpolation simultaneously performs the interpolation and some smoothing of the original data. By nature, through the parameterization of the covariance model, it involves some trade-off between the fidelity to the observed data and the reconstruction of a spatially-consistent field. Hence, interpolated fields typically involve a noticeable smoothing of the truly observed data. The same difficulty arises from the second category of approaches which involves matrix factorization [1], especially EOF-based (Empirical Orthogonal Functions, also referred to as PCA in the image analysis community) approaches, which relies on the projection onto a precomputed basis functions. By contrast, we develop here a different point of view. We rather aim at reconstructing or emulating a spatially- and geophysically-consistent field with a strong fidelity to the observed data. This is referred to as image inpainting in the image processing literature [10], [22]. This is also viewed as a mean to truly handle multi-source data acquired at different resolutions. Model-based interpolation, such as polynomial or spline-based interpolation, may be regarded as possible solutions [38], [40], but they cannot deal with textural patterns.

In the image processing field, image inpainting and super-resolution have been particularly active research topics over the last decade [10], [14], [16], [31], [32]. A variety of models and algorithms have been introduced, including 
for instance variational, Bayesian, non-local setting. As geophysical fields may be viewed as textured images, we focus on texture-related models. Among others, we may distinguish two categories of approaches: exemplar-based or patch-based models and explicit statistical models. Exemplar-based schemes state the inpainting and super-resolution issue as the sampling from a collection of local patches (also referred to as a patch dictionnary). From the original work of Efros et al. [12], the formulation of these exemplar-based strategies as non-local models [5], [29], [32] has become the state-of-art for a wide range of inverse problems in image processing. Applications to remote sensing data have also been investigated [11], including missing data interpolation [24], [39]. By contrast, explicit statistical models rely on specific statistical features such as spatial covariances (or equivalently, spectral power densities) [15], [7], marginal distributions [18], scale-invariance [9]. Recently, the coupling of patch-based representation and of spectral characteristics has been investigated for texture synthesis.

Regarding ocean surface geophysical fields, such statistical features are particularly relevant as ocean turbulence is expected to resort in specific spectral and non-Gaussian characteristics. Besides, for any given case study region, existing image time series provide the mean for building representative dictionaries of exemplars. Therefore, the combination of non-local models and statistical priors, which has not been investigated in previous work to our knowledge, appears as a natural paradigm to address sea surface field reconstruction from multi-source and multiscale satellite data. This work aims at addressing this issue and our main contributions are two-fold:

- the combination of non-local and statistical priors within a mathematically-sound formulation for the texturebased interpolation of geophysical fields;

- the development and evaluation of non-local models for the joint interpolation of multi-source/multi-resolution SST data.

We detail in the subsequent these contributions.

\section{PROPOSED APPROACH}

Formally, the considered missing data interpolation is stated as an inverse problem, i.e. the reconstruction of unknown fields $X^{(1)}$ and $X^{(2)}$ from partial observations $Y^{(1)}$ and $Y^{(2)}$, where the subscripts refers to satellitederived gridded fields from two different sensors. These sensors may differ both in the associated spatial resolution and in the missing data rate. We consider all fields are gridded and interpolated on some reference grid $\Omega$, referred to hereafter as the high-resolution grid. We denote respectively by $\Omega^{(1)}$ and $\Omega^{(2)}$ the missing data region for each sensor, and, $r^{(1)}$ and $r^{(2)}$ the actual resolution associated with each sensor. Subscript (1) refers to the sensor with the lowest resolution. By convention, we assume below for the sake of simplicity that $r^{(1)} \geq 1$ and $r^{(2)}=1$. This amounts to considering below that all scales are defined up to the actual resolution grid.

In this section, we describe the proposed framework as follows. We first introduce the considered multi-resolution representation. Second, we introduce non-local and patch-based priors. Third, the considered statistical priors are detailed. Fourth, we present the proposed interpolation model along with the associated numerical scheme. 


\section{A. Multi-resolution representation}

With a view to handling information sources at different resolutions, we consider a wavelet decomposition of the analysed fields [26]. It comes to decompose field $X$ over an orthonormal basis of functions $\left\{\Psi_{s, i}\right\}$, which are scaled and translated versions of a mother wavelet $\Phi($.$) :$

$$
\forall p \in \Omega, X(p)=\sum_{s, i} X_{s, i} \Psi_{s, i}(p)
$$

where $\left\{X_{s, i}\right\}$ are the wavelet coefficients for field $X$. Indices $s$ and $i$ refer respectively to a scale index and to a spatial position at scale $s$. We consider here a dyadic decomposition, such that scale indices are of the form $2^{j}$. For a given field, the computation of the associated wavelet coefficients are issued from the projections onto the basis functions, which are implemented as decimation and filtering steps iterated from the fine scale to the coarsest scale. It may be noticed that the wavelet decomposition guarantees the exact reconstruction of field $X$ from its wavelet coefficients $\left\{X_{s, i}\right\}$. We let the reader refer to [26] for further details on the wavelet decomposition. We introduce the associated wavelet-based projection operator $\mathcal{P}_{W}^{s^{*} 1}$ for scale $s^{*}$, which refers to the reconstruction of field $X$ from all scales greater than $s *$

$$
\forall p \in \Omega, \mathcal{P}_{W}^{s^{*}}(X)(p)=\sum_{s>s^{*}, i} X_{s, i} \Psi_{s, i}(p)
$$

It may be noted that, by construction, field $X-\mathcal{P}_{W}^{s^{*}}(X)$ refers to a detail field, denoted by $\delta \mathcal{P}_{W}^{s^{*}}(X)$ which only involves information at scales finer than $s^{*}$.

For the targeted issue, we denote by $s_{L R}$ the reference low-resolution scale in the dyadic wavelet decomposition, given as the dyadic scale $\left\lceil\log _{2} r^{(1)}\right\rceil$ the closest to the low resolution $r^{(1)}{ }^{2}$. Given that the two sensors observe the same geophysical condition, we assume that the two fields $X^{(1)}$ and $X^{(2)}$ share the same low-resolution projection at scale $s^{L R}$ such that we impose

$$
\mathcal{P}_{W}^{s_{L R}}\left(X^{(1)}\right)=\mathcal{P}_{W}^{s_{L R}}\left(X^{(2)}\right)
$$

\section{B. Multimodal patch-based interpolation and non-local projection}

As stressed in the introduction, patch-based and non-local priors are particularly appealing to develop data-driven image processing techniques, by contrast to model-driven techniques [11], [14], [24]. This appears especially relevant when the definition of explicit analytical a priori models is a complex issue, as for sea surface turbulent dynamics whose characteristics may vary in space and time. We here focus on non-local regularization or projection [5], [29], [32]. Given a field $X$, it comes to compute a non-local mean $\mathcal{P}_{N L M}\left(\left\{\mathcal{A}_{k}\right\}\right)(X)$, with respect to a reference set of patches based on patch similarities. The non-local mean for a patch around a pixel $p \in \Omega$ is given by

$$
\mathcal{P}_{N L M}\left(\left\{\mathcal{A}_{k}\right\}\right)(X)(p)=\sum_{k \in \mathcal{K}} w_{k}\left(X,\left\{\mathcal{A}_{l}\right\}, p\right) \mathcal{A}_{k}
$$

\footnotetext{
${ }^{1}$ By convention, throughout the manuscript, notation $\mathcal{P}$ refers to a projection operator in the image domain. Here, notation $\mathcal{P}_{W}$ refers to a wavelet-based projection operator. In the subsequent, we introduce other projection operators corresponding to the non-local, covariance-based and margin-based priors.

${ }^{2}\lceil$.$\rceil is the ceiling function, such that \lceil a\rceil$ is the integer part of number $a$ plus one.
} 
where $k$ is a patch index within the reference patch dictionary, $\mathcal{A}_{k}$ the $k^{t h} P \times P$ patch in this dictionary. Typical values for the patch width $P$ are between 5 and 13. $w_{k}\left(I, \mathcal{A}_{k}, p\right)$ is a weighing factor which weighs the contribution of the $\mathrm{k}^{t h}$ reference patch $\mathcal{A}_{k}$ in the non-local mean at point $p$. It relies on the similarity between the $P \times P$ patch around pixel $p$ in field $X$, denoted by $X\left(\mathcal{N}_{p}\right)$, and reference patch $\mathcal{A}_{k}$. Patch similarities are generally evaluated according to a classical Euclidean distance [5], [32], such weight $w_{k}\left(X,\left\{\mathcal{A}_{l}\right\}, p\right)$ resorts to a normalised weight as follows:

$$
w_{k}\left(X,\left\{\mathcal{A}_{l}\right\}, p\right)=\frac{g\left(\left\|X\left(\mathcal{N}_{p}\right)-\mathcal{A}_{k}\right\|\right)}{\sum_{l} g\left(\left\|X\left(\mathcal{N}_{p}\right)-\mathcal{A}_{l}\right\|\right)}
$$

where $g($.$) is a kernel function. Non-local regularisation [5], [29], [32] typically considers a Gaussian kernel$ $g(x)=\exp \left(-\gamma \cdot x^{2}\right)$ with $\gamma$ a smoothing parameter to be defined. In texture synthesis, nearest-neighbor kernels may be preferred to avoid some over smoothing of textured details. Different strategies may also be considered for the combination of the non-local means computed for different image positions. Non-local denoising and segmentation [5], [32] typically involves the computation of the non-local mean for every pixel to derive the non-local image projection. By contrast, in texture synthesis and inpainting [10], [14], [22], one usually achieves the non-local projection for partially or non-overlapping patches with a predefined, optimized or randomized selection of the pixels at which the non-local means are actually evaluated (Eq.4). The later, which is the more generic, is selected in this work. We apply the non-local means to randomly selected positions and, for each selected position $p$, we update the non-local image projection with the $(P-B) \times(P-B)$ interior of the non-local mean $P \times P$ patch $(4)$ with $B$ the width at the boundary. It may noted that, in our implementation, we use for each non-local projection a different random sequence of patch positions with a view to ensuring shift invariance [26].

Here, we apply the non-local setting to the joint interpolation of the two fields $X^{(1)}$ and $X^{(2)}$ associated with two different sensors. Hence, the evaluation of patch similarities naturally relies on the information from each field and the reference patch dictionary $\left\{\mathcal{A}_{k}^{(1)}, \mathcal{A}_{k}^{(2)}\right\}$ is formed by concomitant patch exemplars form each sensor. Non-local projection (4) becomes a multi-modal non-local projection. For the sake of simplicity and readability, we drop the explicit reference to patch dictionary $\left\{\mathcal{A}_{k}^{(1)}, \mathcal{A}_{k}^{(2)}\right\}$ in our notations. For field $X^{(1)}$, we denote by $\mathcal{P}_{N L M, X(2)}\left(X^{(1)}\right)$ its non-local projection given reference patch dictionary $\left\{\mathcal{A}_{l}^{(1)}, \mathcal{A}_{l}^{(2)}\right\}$ and field $X^{(1)}: \forall p \in \Omega$

$$
\mathcal{P}_{N L M, X^{(2)}}\left(X^{(1)}\right)\left(\mathcal{N}_{p}\right)=\sum_{k \in \mathcal{K}} w_{k}\left(X^{(1)}, X^{(2)}, p\right) \mathcal{A}_{k}^{(1)}
$$

where the normalized non-local weights involve joint similarities for the two fields

$$
\begin{aligned}
w_{k}\left(X^{(1)}, X^{(2)}, p\right) \propto \quad & g\left(\alpha^{(1)}\left\|X^{(1)}\left(\mathcal{N}_{p}\right)-\mathcal{A}_{k}^{(1)}\right\|\right. \\
& \left.+\alpha^{(2)}\left\|X^{(2)}\left(\mathcal{N}_{p}\right)-\mathcal{A}_{k}^{(2)}\right\|\right)
\end{aligned}
$$

where $\alpha^{(1)}$ and $\alpha^{(2)}$ are scalar values which weigh the relative contribution of each sensor. Regarding the targeted issue, we explore different strategies for the multi-modal non-local projection:

- Projection $\mathcal{P}_{N L M, X^{(2)}}\left(X^{(1)}\right)$ : the non-local projection of field $X^{(1)}$ given field $X^{(2)}$; 
- Projection $\mathcal{P}_{N L M,\left\|\nabla X^{(2)}\right\|}\left(\delta X^{(1)}\right)$ : the non-local projection of detail field $\delta X^{(1)}$ given field $\left\|\nabla X^{(2)}\right\|$. This strategy is motivated by recent observations that the variability of the high-resolution detail $\delta X^{(1)}$ is driven by the magnitude of the low-resolution gradient $\left\|\nabla X^{(2)}\right\|[13]$.

In the reported experiments, we compare these different strategies and evaluate their pros and cons.

\section{Statistical constraints}

Geophysical fields are expected to depict specific statistical features [37], [20], [4], [6], which may be used as additional priors to further constrain their reconstruction from partial and noisy observations. In this work, we focus on two categories of statistical features: covariance (or equivalently spectral) features and non-Gaussianity.

Sea surface geophysical processes, especially for the mesoscale range (i.e., for horizontal scales from tens of kilometres to hundreds of kilometres), involve turbulent processes characterised by specific spectral signatures [20], [25]. Their radial power spectral density generally involves power laws [37], [20]. A typical example is the homogeneous and isotropic turbulence for incompressible flows [19] which resorts to Kolmogorov's $5 / 3$ power law for the power spectral density of the energy of the velocity field. We then set a power law prior on the radial spectral density of geophysical fields $X^{(1)}$ and $X^{(2)}$ for the fine-scale range (i.e., scales lower than $r^{(1)}$ ). Let us denote by $R^{*}$ the reference radial power spectral density and $S_{X, X}$ the power spectral density of field $X$. The radial power spectral density of field $X$, denoted by $R_{X}$ is a radial integration of power spectral density $S_{X, X}$ :

$$
R_{X}(r)=\int_{\|\omega\|=r} S_{X, X}(w) d w
$$

It may be noted that power spectral density $S_{X, X}$ is the Fourier transform of the spatial covariance of field $X$. The considered spectral prior comes to a define a reference radial power spectral density $R^{*}$. In the reported numerical experiments, we consider both a non-paramatric densities and power-law parameterization as:

$$
R^{*}(r)=\beta \cdot r^{\xi}
$$

where $\gamma$ and $\alpha$ are the parameters of this spectral prior. In the reported experiments, we calibrate these parameters

from the spatial covariance of field $X^{(1)}$ for scales greater than $r^{(1)}$ (typically, greater than $100 \mathrm{~km}$ for the considered experiments on SST data). Given this prior, the fine-scale spectral constraint for field $X^{(2)}$ amounts to:

$$
R_{X(2)}(r)=R^{*}(r), \forall r \in\left[r^{(2)}, r^{(1)}\right]
$$

This spectral constraint can be solved for in the Fourier domain through the computation of a transformed field $\mathcal{T}_{R_{r(1)}^{*}}(X)$ such that its Fourier transform $\mathcal{T}_{R_{r(1)}^{*}}(X)$ is given by:

$$
\widehat{\mathcal{T}_{R^{*}}(X)}=\sqrt{\frac{R^{*}}{R_{X}}} \widehat{X}
$$

Numerically, the Fourier transform is computed here using a windowed FFT. It might be pointed out that the Fourier transform amounts to a linear filtering and a projection onto the manifold of the two-dimensional fields accounting for spectral constraint (10). 
In addition to this spectral constraint, ocean turbulence is widely acknowledged to depict non-Gaussian marginals in multiscale decomposition [6], [13], [27] as considered here. Turbulent flows tend to advect tracers to form fronts or high-gradient structures at different scales. This in turn results in non-Gaussian heavy-tailed marginals of the detail fields in the considered multi-scale representation. Generalized Gaussian distributions $\mathcal{G G}_{\sigma, \alpha}$ appear as relevant, yet simple, parametric models to account for the observed non-Gaussian signatures:

$$
\mathcal{G G}_{\sigma, \alpha}(x) \propto \exp \left(-\left(\frac{x}{2 \sigma}\right)^{\nu}\right)
$$

where $\sigma$ is a scaling parameter and $\nu$ a power exponent. For $\nu=2$, it resorts to a standard zero-mean Gaussian distribution. For a given field $X^{(2)}$, we fit a generalized Gaussian distribution to the detail field $\delta X^{(2)}-\mathcal{P}_{W}^{s^{L R}}\left(X^{(2)}\right)$ according to a maximum likelihood (ML) criterion. Let us denote by $\mathcal{G G}_{\sigma^{*}, \alpha^{*}}$ the reference distribution. The marginal constraint then amounts to:

$$
X^{(2)}-\mathcal{P}_{W}^{s_{L R}}\left(X^{(2)}\right) \propto \mathcal{G} \mathcal{G}_{\sigma^{*}, \alpha^{*}}
$$

To solve for this constraint for field $X^{(2)}$, we proceed as follows. Given current fields $X^{(1)}$ and $X^{(2)}$, we fit a generalized Gaussian distribution $\mathcal{G G}_{\tilde{\sigma}, \tilde{\alpha}}$ to the current detail field $X^{(2)}-X^{(1)}$ according to a ML criterion. The transformed field $\mathcal{T}_{\mathcal{G}} \mathcal{G}^{*}\left(X^{(2)}\right)$ resorts to:

$$
\begin{aligned}
\mathcal{T}_{\mathcal{G} \mathcal{G}^{*}}(X) & =\mathcal{P}_{W}^{s_{L R}}\left(X^{(2)}\right) \\
& +\Psi_{\sigma^{*}, \alpha^{*}}\left(\Psi_{\tilde{\sigma}, \tilde{\alpha}}^{-1}\left(X^{(2)}(p)-\mathcal{P}_{W}^{s_{L R}}\left(X^{(2)}\right)(p)\right)\right)
\end{aligned}
$$

where $\Psi_{\sigma, \alpha}$ is the cumulative distribution function of generalized Gaussian distribution $\mathcal{G G}_{\tilde{\sigma}, \tilde{\alpha}}, \Psi_{\tilde{\sigma}, \tilde{\alpha}}^{-1}$ the associated inverse cumulative distribution function.

\section{Joint interpolation and blending framework}

The joint interpolation and blending of two fields $X^{(1)}$ and $X^{(2)}$ from partial observations $Y^{(1)}$ and $Y^{(2)}$ is stated as the following joint non-local projection:

$$
\left\{\begin{array}{l}
\widehat{X^{(1)}}=\arg \min _{X^{(1)}}\left\|X^{(1)}-\mathcal{P}_{N L M, X^{(2)}}\left(X^{(1)}\right)\right\|_{\Omega^{(1)}}^{2} \\
\widehat{X^{(2)}}=\arg \min _{X^{(2)}}\left\|X^{(2)}-\mathcal{P}_{N L M, X^{(1)}}\left(X^{(2)}\right)\right\|_{\Omega^{(2)}}^{2}
\end{array}\right.
$$


where $\|\cdot\|_{\Omega}^{2}$ stands for the quadratic norm restricted to spatial domain $\Omega$. The above minimization is to be solved for under the four following constraints:

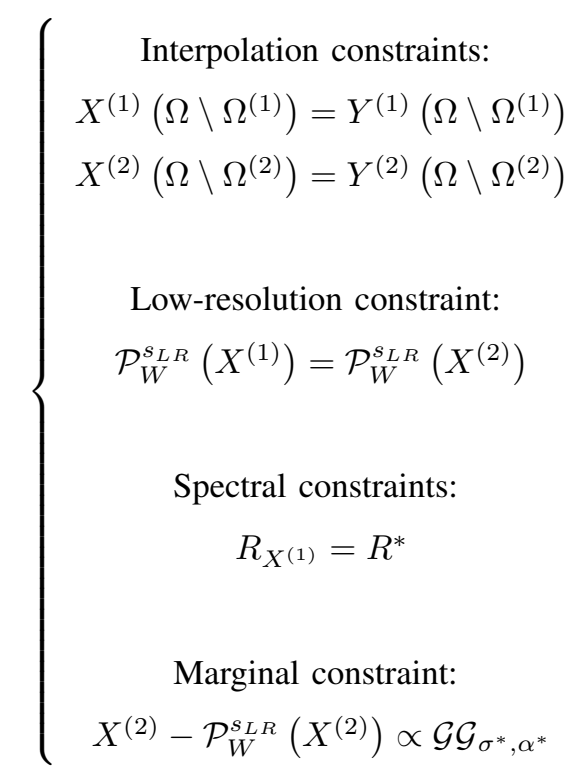

Problem formulation (15) refers to the application of the non-local projections to field $X^{(1)}$ given field $X^{(2)}$. As described in Section III-B, for field $X^{(2)}$, we consider a different strategy with non-local projection $\mathcal{P}_{N L M,\|\nabla X(2)\|}\left(\delta X^{(1)}\right)$ applied to the detail field. This formulation refers to the two key contributions of this work, namely:

- the combination of non-local priors to statistical priors through both non-local projections (15) and spectral and marginal constraints in (16);

- the application of non-local models for the joint interpolation of multi-source/multi-resolution SST data highlighted by both interpolation constraints in (16) and non-local projections (15) at low- and high-resolution.

We summarize in Table?? in the Supplementary material the different variables and parameters considered in this study.

\begin{tabular}{|c|c|c|}
\hline \hline Symbol & Description & Default value \\
\hline \hline$\gamma$ & Scale parameter of the Gaussian kernel, Eq.(5) & 1 \\
$\alpha^{(1)}, \alpha^{(2)}$ & Relative weights of modality $(i)$ in the non-local weight, Eq.(7) & 0.5 \\
$\beta, \xi$ & Parameters of the spectral prior, Eq.(9)t & - \\
$\sigma, \nu$ & Parameters of the prior on the marginal of the details, Eq.(12) & - \\
$\lambda_{I}, \lambda_{M}, \lambda_{S}$ & Gradient descent coefficients for the interpolation, marginal and spectral constraints, Algorithms 2\& 3 & 0.1 \\
\hline \hline
\end{tabular}

SUMMARY OF THE PARAMETERS CONSIDERED IN OUR MODEL

To solve for this constrained minimization, we consider an iterative procedure. From given initialization $X^{(1), 0}$ and $X^{(2), 0}$, it comes to iterate the non-local projections of the current solutions $X^{(1), n}$ and $X^{(2), n}$ and the projection of the resulting fields $\mathcal{P}_{N L M, X^{(2)}}\left(X^{(1), k}\right)$ and $\mathcal{P}_{N L M, X^{(1)}}\left(X^{(2), k}\right)$ onto the four additional constraints (16). Numerically, this approach involves three key components: 
- The projection onto interpolation and low-resolution constraints (first-two constraints in (16)): we solve for this projection using a gradient-based descent to retrieve the field accounting for the interpolation constraint while minimizing the quadratic norm to the expected low-resolution field (see Algorithm (1) in the Supplementary Material for details);

- The projection onto the constraints on the spectral and marginal constraints. Based on transforms (14) and (11), we use a gradient-based descent to jointly solve for these two constraints. Each iteration of this gradientbased descent involves a projection onto interpolation and low-resolution constraints (see Algorithm (2) in Supplementary Material for details);

- The overall algorithm iterates three main steps: i) non-local projections of fields $X^{(1)}$ and $X^{(2)}$ followed by a projection onto interpolation and low-resolution constraints, ii) projections of resulting field updates onto the marginal and spectral constraints, iii) the update of the reference low-resolution field as the mean of the low-resolution projection of the updated fields $X^{(1)}$ and $X^{(2)}$ as well as the update of reference generalised Gaussian distribution $\mathcal{G G}_{\sigma^{*}, \alpha^{*}}$ for the high-resolution details of field $X^{(1)}$ (cf. (13)). The procedure is described in the Supplementary Material by Algorithm (1).

The initialization of fields $X^{(1)}$ and $X^{(2)}$ from partial observations $Y^{(1)}$ and $Y^{(2)}$ may exploit classical interpolation techniques. In the reported experiments, we consider a initial variational interpolation using the AMLE (Absolutely Minimizing Lipschitz Extension) [8]. An additional random component is added in missing data area $\Omega^{(2)}$ for highresolution field $X^{(2)}$. We use a white Gaussian noise, such that initial field $X^{(2)}$ involves the expected variance for high-resolution detail $X^{(2)}-\mathcal{P}_{W}^{s_{L R}}\left(X^{(2)}\right)$.

\section{RESUlts}

We report numerical experiments to evaluate the proposed framework for two types of data: i) high-resolution SST data from ODYSSEA analysis [2] for which we simulate missing data and a low-resolution condition, ii) real AMSR-E and SEVIRI daily SST data.

\section{A. ODYSSEA SST data}

Considered data and expriments: ODYSSEA SST fields are gridded and interpolated composite fields produced by Ifremer/CERSAT [2] onto a grid with a $0.02^{\circ}$ spatial resolution $(\sim 2 \mathrm{~km})$. ODYSSEA analysis combines both microwave sensor data (including TMI and AMSR-E data) and infrared sensor data (including AVHRR, VISSR and SEVIRI data). It involves different processing steps, especially sensor-specific bias correction, sensor intercalibration and optimal interpolation of missing data areas. The selected field (January, 8th 2012) and case study area, off South Africa, are chosen for the the quality of the composite field and the richness of the observed SST structures (e.g., filaments, fronts).

From the considered ODYSSEA SST field, we first evaluate the relevance of the proposed model for the interpolation of a high-resolution field given a known low-resolution condition, i.e. observation $Y^{(1)}$ does not involve missing data and $X^{(1)}=Y^{(1)}$. We proceed as follows. For a given dyadic downsampling factor $\kappa$, here 16 


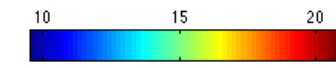

LR initalization
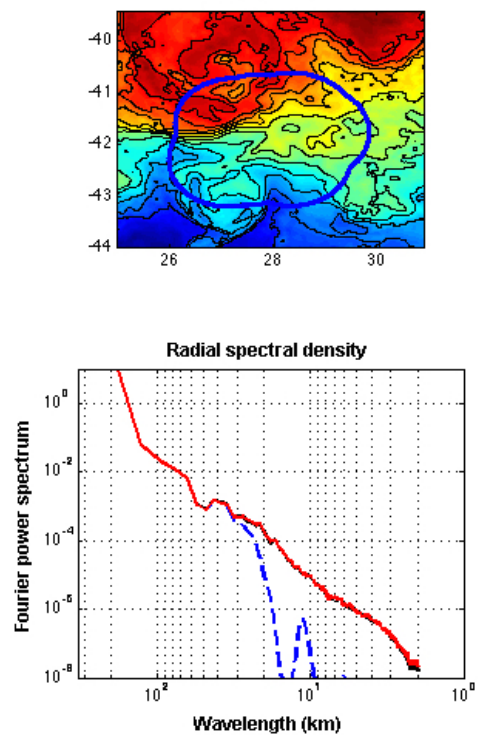

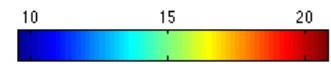

Real HR field
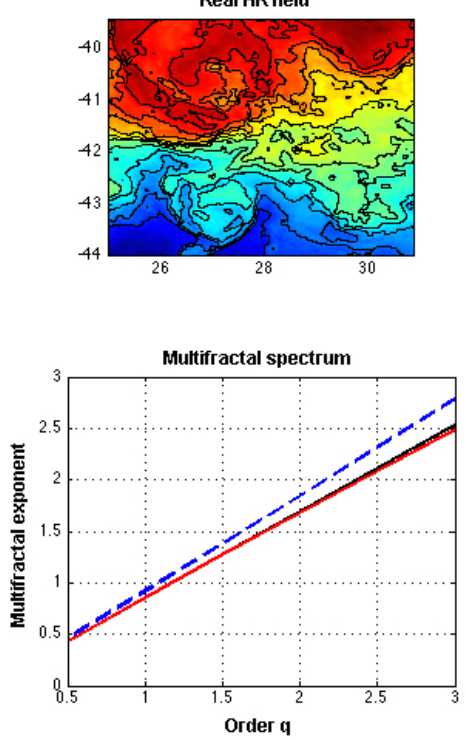

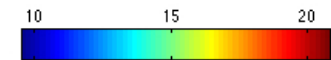

HR Interpolation
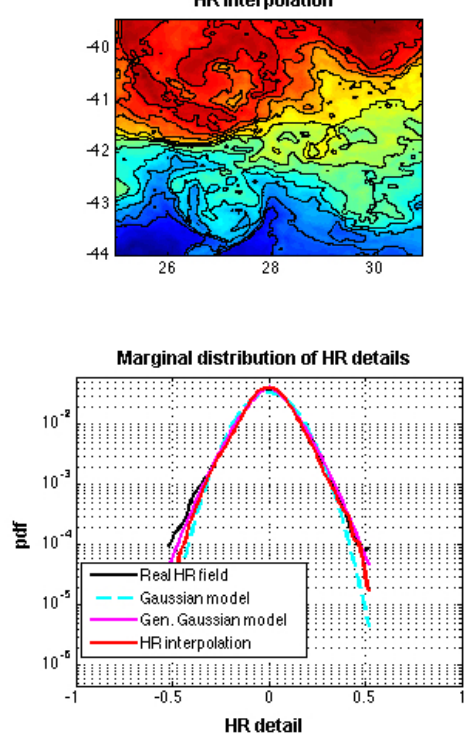

Fig. 1. Interpolation results for a large simulated missing data area: first row, low-resolution SST field (subsampling factor of 16 from a 8-Daubechies wavelet of the ODYSSEA SST field) with superimposed the contour of the missing data area in blue (left), high-resolution ODYSSEA SST field (resolution grid of $2 \mathrm{~km}$ ) (center), interpolated field using the proposed model (right); second row, radial spectral densities of the low-resolution (blue, -), real high-resolution (black,-) and interpolated high-resolution (red, -) fields (left), multifractral spectrum of the low-resolution (blue, -), real (black,-) and interpolated high-resolution fields (center), distribution of high-resolution detail $\delta \mathcal{P}_{W}^{{ }^{L} R}\left(X^{(2)}\right)$ for the real real (black,-) and interpolated (red, -) fields along with the target generalized Gaussian distribution (magenta, -) and a Gaussian distribution with the same variance (cyan,-). We superimpose image level-lines to illustrate the underlying spatial geometry.

(i.e., $r^{(1)}=2 k m$ and $r^{(1)}=32 k m$ and $s^{L R}=4$ ), we derive the low-resolution condition $X^{(1)}$ as $\mathcal{P}_{W}^{s_{L R}}\left(X^{(2)}\right)$ the low-resolution projection of the considered ODYSSEA field with a Daubechies- 8 wavelet transform at scale $s_{L R}=\log _{2}(\kappa)$. Regarding high-resolution observation $Y^{(2)}$, we generate randomly-distributed missing data. With a view to evaluating the performance of the model and the relative importance of the non-local and statistical priors, we may consider a random missing data pattern with large missing data area (as in Fig.1 with a mean radius of 150 pixels ) or small missing data areas (here, 10-pixel-wide missing data areas).

The considered parameter setting for the non-local priors is as follows. We select $500011 \times 11$ patches off the missing data area. The non-local means is computed for the first five neighbors (Eq.7). The scale parameter $\gamma$ of the Gaussian kernel is set to 1 (Eq.5) and relative weights for $\alpha^{(1)}$ and $\alpha^{(2)}$ (Eq.7) are set to 0.5 and 0.5 for non-local projection $\mathcal{P}_{N L M, X^{(2)}}\left(X^{(1)}\right)$, and to 0.1 and 0.9 for non-local projection $\mathcal{P}_{N L M,\left\|\nabla X^{(2)}\right\|}\left(\delta X^{(1)}\right)$. The reference power spectral density $R^{*}$ is the radial spectral density of the high-resolution field. Similarly, the reference marginal distribution $\mathcal{G G}_{\sigma^{*}, \alpha^{*}}$ is the generalized Gaussian distribution fitted to the detail of the true high-resolution field according to a Maximum Likelihood criterion $\left(\sigma^{*}=0.08\right.$ and $\left.\alpha^{*}=1.44\right)$.

Comparison of stochastic interpolation models: We report in Fig.1 the interpolation results for the large missing 

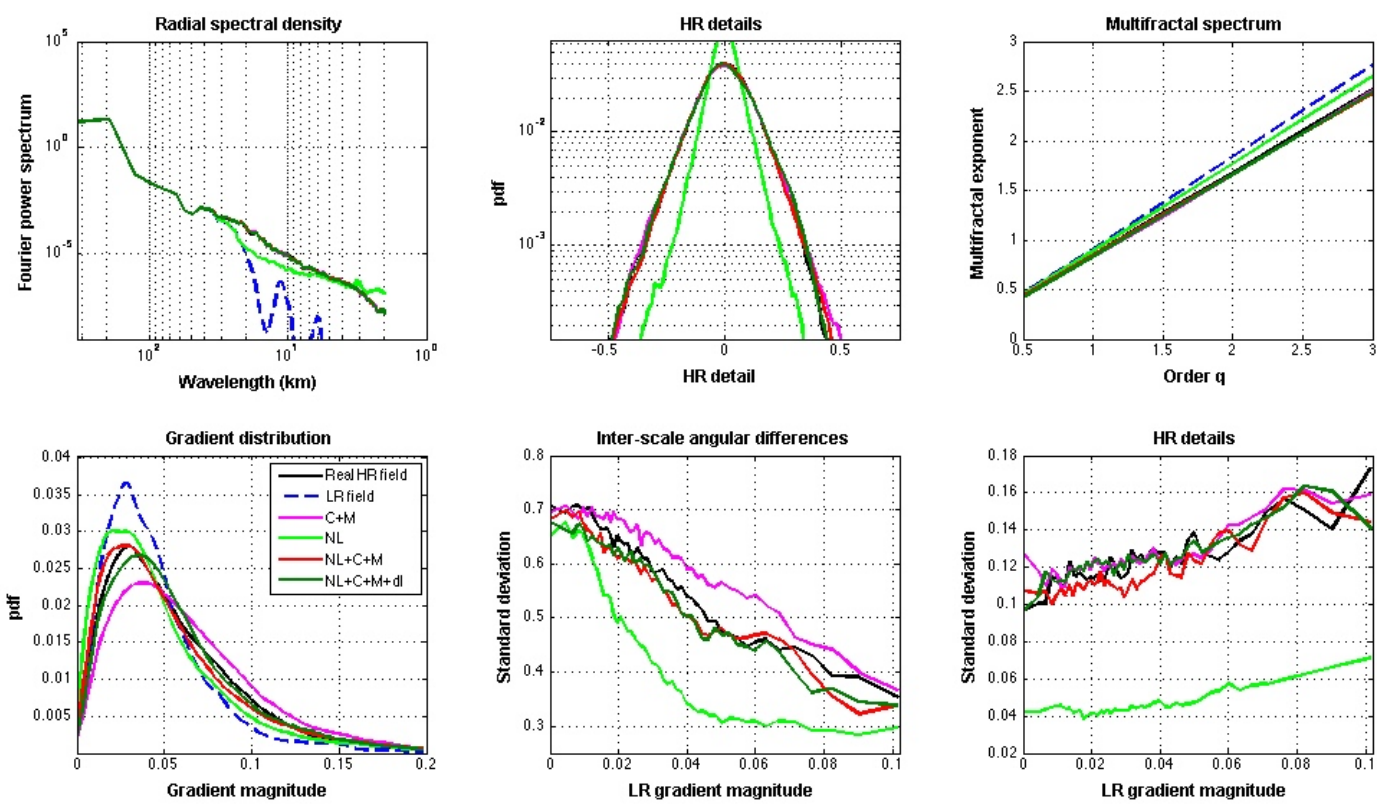

Fig. 2. Statistical analysis of interpolation results for the synthetic case considered in Fig.1: first row, radial spectral density (left), multifractal spectrum (center), distribution of high-resolution detail $\delta \mathcal{P}_{W}^{L R}\left(X^{(2)}\right)$ (right); second row, distribution of the norm of the gradient $\|\nabla X\|$ (left), standard deviation of high-resolution detail $\delta \mathcal{P}_{W}^{s^{L} R}\left(X^{(2)}\right)$ a a function of the low-resolution gradient $\left\|\nabla X^{(1)}\right\|$ (center), standard deviation of the angular difference between gradient fields $\nabla X^{(1)}$ and $\nabla X^{(2)}$ as a function of the low-resolution gradient $\left\|\nabla X^{(1)}\right\|$ (right). For each subplot, we report the features associated with the real high-resolution field (black,-), the low-resolution field (blue,-), and four different interpolation models, namely using only the covariance and marginal constraints ( $\mathrm{C}+\mathrm{M}$, magenta,-), using only the non-local projection (NL, light green, -), using the proposed model $(\mathrm{NL}+\mathrm{C}+\mathrm{M}$, red,-), using the proposed model with the non-local projection applied to detail field $\delta \mathcal{P}_{W}^{s^{L R}}\left(X^{(2)}\right)$ rather than field $X^{(2)}(\mathrm{NL}+\mathrm{C}+\mathrm{M}+\mathrm{dX}$, dark green,-).

area example. These interpolation results are issued from the non-local projection of field $X^{(2)}$ given low-resolution field $X^{(1)}$. As illustrated, the interpolated field depict to the expected spectral and marginal characteristics. With a view to evaluating the relative importance of the non-local an statistical priors, we consider four different texturebased interpolation models:

- a model relying only on the spectral and marginal priors (model $C+M)^{3}$;

- a model using only the non-local prior (model $N L)^{4}$;

- the proposed model combining the non-local and statistical priors (model $N L+C+M$ );

- the proposed model combining the non-local and statistical priors with the non-local projection applied to detail field $\delta \mathcal{P}_{W}^{s^{L R}}\left(X^{(2)}\right)$ rather than field $X^{(2)}$ (model $\left.N L+C+d X\right)$.

\footnotetext{
${ }^{3}$ This comes to apply Algorithm (1) in the Supplementary Material without the non-local projection step.

${ }^{4}$ This comes to apply Algorithm (1) in the Supplementary Material without projections onto the spectral and marginal constraints.
} 

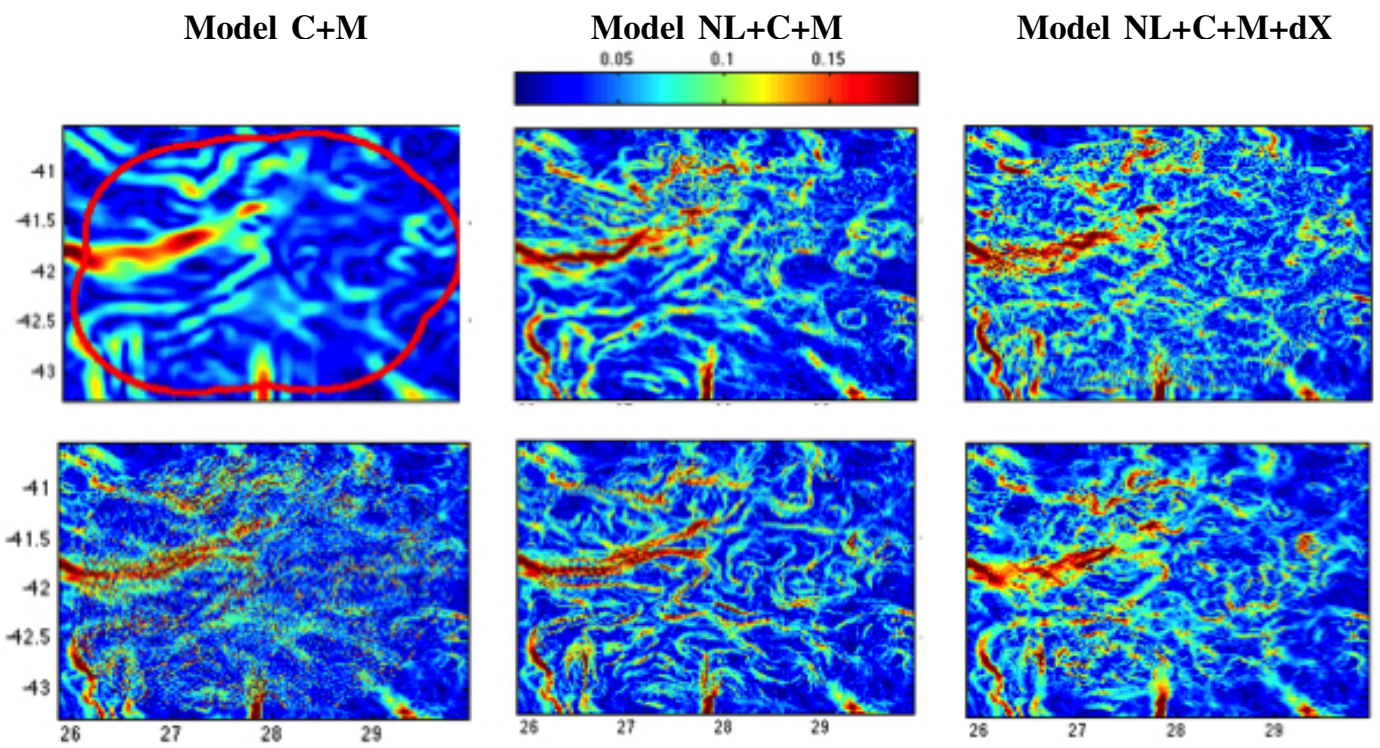

Fig. 3. Gradient fields of the interpolation compared in Fig.2: from left to right and top to bottom, low-resolution field with superimposed the contour of the missing data area, reference high-resolution field and four different interpolation models, namely using only the covariance and marginal constraints $(\mathrm{C}+\mathrm{M})$, using only the non-local projection $(\mathrm{NL}+\mathrm{C}+\mathrm{M})$, using the proposed model, using the proposed model with the non-local projection applied to detail field $\delta \mathcal{P}_{W}^{s^{L R}}\left(X^{(2)}\right)$ rather than field $X^{(2)}(\mathrm{NL}+\mathrm{C}+\mathrm{M}+\mathrm{dX})$.

As depicted in Fig.2, we perform a quantitative comparison of these four models according to six statistical features ${ }^{5}$ : the radial spectral density, the multifractal spectrum, the marginal distribution of high-resolution details $\mathcal{P}_{W}^{s_{L R}}\left(X^{(2)}\right)$, the distribution of the magnitude of gradient $\nabla X^{(2)}$, the standard deviation of high-resolution detail $\mathcal{P}_{W}^{s_{L R}}\left(X^{(2)}\right)$ as a function of the norm of low-resolution gradient $\nabla X^{(1)}$ and the standard deviation of the angular differences between low-resolution and high-resolution gradients as a function of the norm of low-resolution gradient $\nabla X^{(1)}$. These different features are selected to analyse the extent to which we recover the inter-scale characteristics of the reference field. These results clearly stress the relevance of the combination of a non-local prior with covariance and marginal priors. The sole use of a non-local prior leads to high-resolution field which depicts too much fine-scale energy. The combination with the covariance-based prior allows us to further regularize the non-local projections and to match the expected spectral properties. It also leads to a better fit in terms of gradient statistics and inter-scale angular statistics, as the non-local prior exploits an actual conditioning of the high-resolution detail from the lowresolution field. This is further illustrated by the visual inspection of the gradient field of the different interpolation results (Fig.3). Little difference is observed between the proposed model whether we apply the non-local projection to high-resolution field $X^{(2)}$ or to high-resolution detail field $\mathcal{P}_{W}^{s_{L R}}\left(X^{(2)}\right)$.

Comparison to a kriging-based interpolation: We report a comparison Fig.4 to a kriging-based interpolation

\footnotetext{
${ }^{5}$ It might be noted that classical mean square error (MSE) statistics do not apply for texture-based interpolation and super-resolution for large subsampling factors [9], [14] as considered here. For textured fields, small spatial translations of the high-resolution details generate large MSE values, so that the interpolant, which minimizes the MSE would resort to a non-textured field as illustrated in Fig.4 with a kriging-based interpolation
} 
for the example considered in Fig.1. We apply the kriging-based interpolation to the detailed field $\mathcal{P}_{W}^{s_{L R}}\left(X^{(2)}\right)$. The considered covariance model is a stationary isotropic Matern model fitted to the true detailed field $\mathcal{P}_{W}^{s_{L R}}\left(X^{(2)}\right)$ [33]. We resort to a Matern covariance with a standard deviation of 0.01 , a power exponent of 3 and a spatial range of 6 pixels. Under a Gaussian hypothesis, kriging-based interpolation comes to derive a linear prediction of the missing data from the truly observed ones. This linear prediction refers to the mean of the conditional Gaussian distribution of the the missing data given the truly observed ones. This mean value then minimizes the mean square reconstruction error. For points, which are far from any truly observed data, the linear prediction converges to the mean of the Gaussian process, that is to say that, in terms of mean square error statistics, the best prediction is the mean of the process is no close-enough points is truly observed. This is observed in Fig.4. Given the spatial range of the fitted covariance model, the linear prediction of the high-resoluton detail for points which are further than 10 pixels from the boundary of the missing data area is close to 0 , so that the kriging-based interpolation resorts to a relative mean square error close to 1 , which means it cannot bring additional information compared to the low-resolution field. As such, the interpolated field does not depict spectral and statistical patterns similar to the true high-resolution field (Fig.5). This is intrinsically different from the considered interpolation strategy. We do not aim at minimizing a mean square reconstruction error statistics and retrieve a relative mean square error of 1.3 larger than the kriging-based interpolation due to small spatial misalignements between the true and simulated high-resolution details. Meanwhile, as shown in (Fig.4 \& Fig.5), we emulate a spatially-consistent field, where the high-resolution details are statistically-consistent at both observed and missing data points. Similar experiments have been performed for random small missing areas with a mean radius of 10 pixels, for which little difference is reported between the kriging-based and proposed interpolation models both in terms of mean square error statistics (0.61 vs. 0.67) and of the statistical features reported in Fig.5.

\section{B. Joint analysis of AMSR-E and SEVIRI SST data}

We consider an application of the proposed framework to the joint analysis of AMSR-E and SEVIRI SST fields. AMSR-E SST field involves a typical resolution grid of $0.2^{\circ}$, whereas we consider daily composite SEVIRI field with a $0.1^{\circ}$ grid resolution. From Fig.6, one can notice that their radial power spectral density differ from about $1^{\circ}$, with a significantly lower spectral energy of the AMSR-E field. This difference relates to the interpolation and filtering applied to AMSR-E fields. The situation reported in Fig.6 depicts a low missing data rate for the AMSR-E field, but a much higher missing data rate, especially off South Africa for the SEVIRI field. Besides, due to the associated geostationary orbit, no SEVIRI data is available, whatever the atmospheric conditions, for the extreme south-east area of the case-study region. In a similar way, contrary to SEVIRI data, AMSR-E data do not provide any information close to the sea shore. Regarding the parameterization of the proposed model, we proceed as follows. For the reference radial power spectral density, we consider a power law fitted for scales $\left[0.8^{\circ}, 3^{\circ}\right]$ and extrapolated to the finer scales. Here, in Algorithm (??), the spectral constraint is applied globally and not only in the missing data area with a view to filtering noisy patterns observed in the daily SEVIRI composite. As patch dataset, we randomly sample joint patches in the observed AMSR-E and SEVIRI fields for the considered data and 

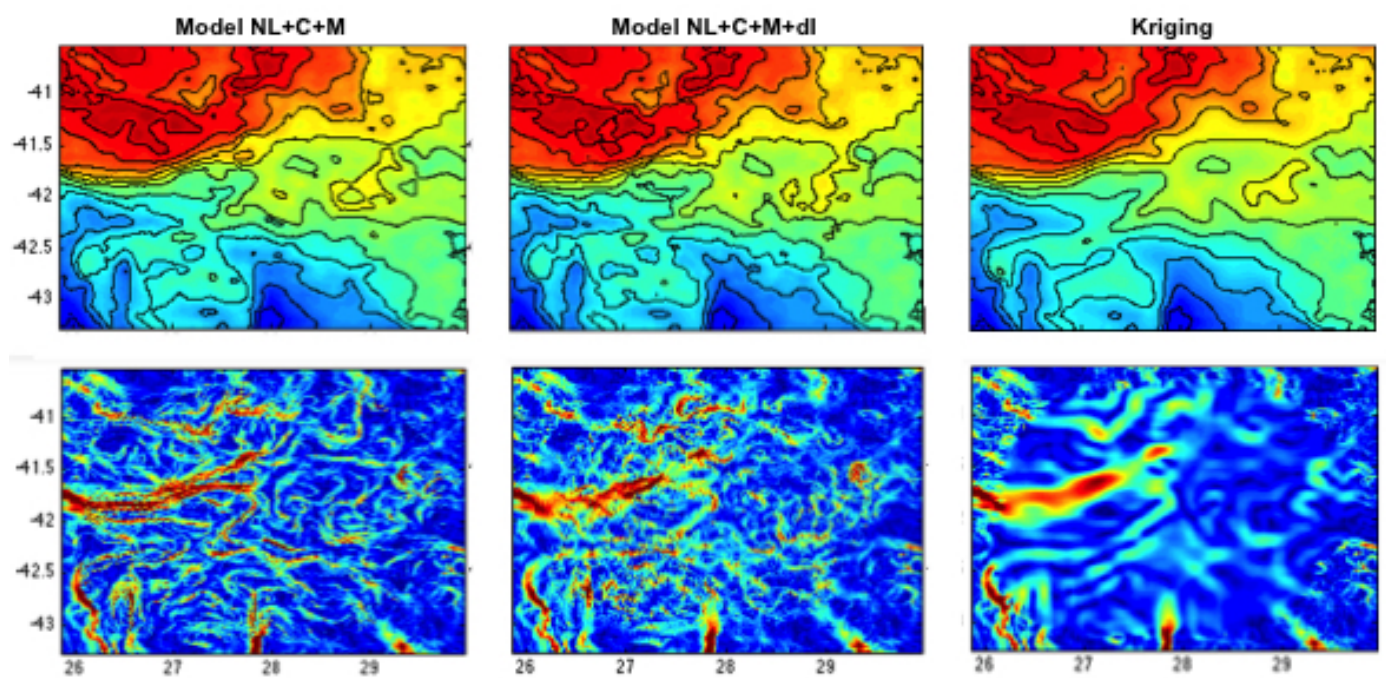

Fig. 4. Visual comparison to a kriging-based interpolation for the example considered in Fig.1: first row, interpolated fields; second row, associated gradient magnitude fields. We report results using the proposed model (model NL+C+M, left), the proposed model with the non-local projection applied to detail field $\delta \mathcal{P}_{W}^{s^{L R}}\left(X^{(2)}\right)$ (model $\mathrm{NL}+\mathrm{C}+\mathrm{M}+\mathrm{dX}$, center) and a kriging-based model (right).
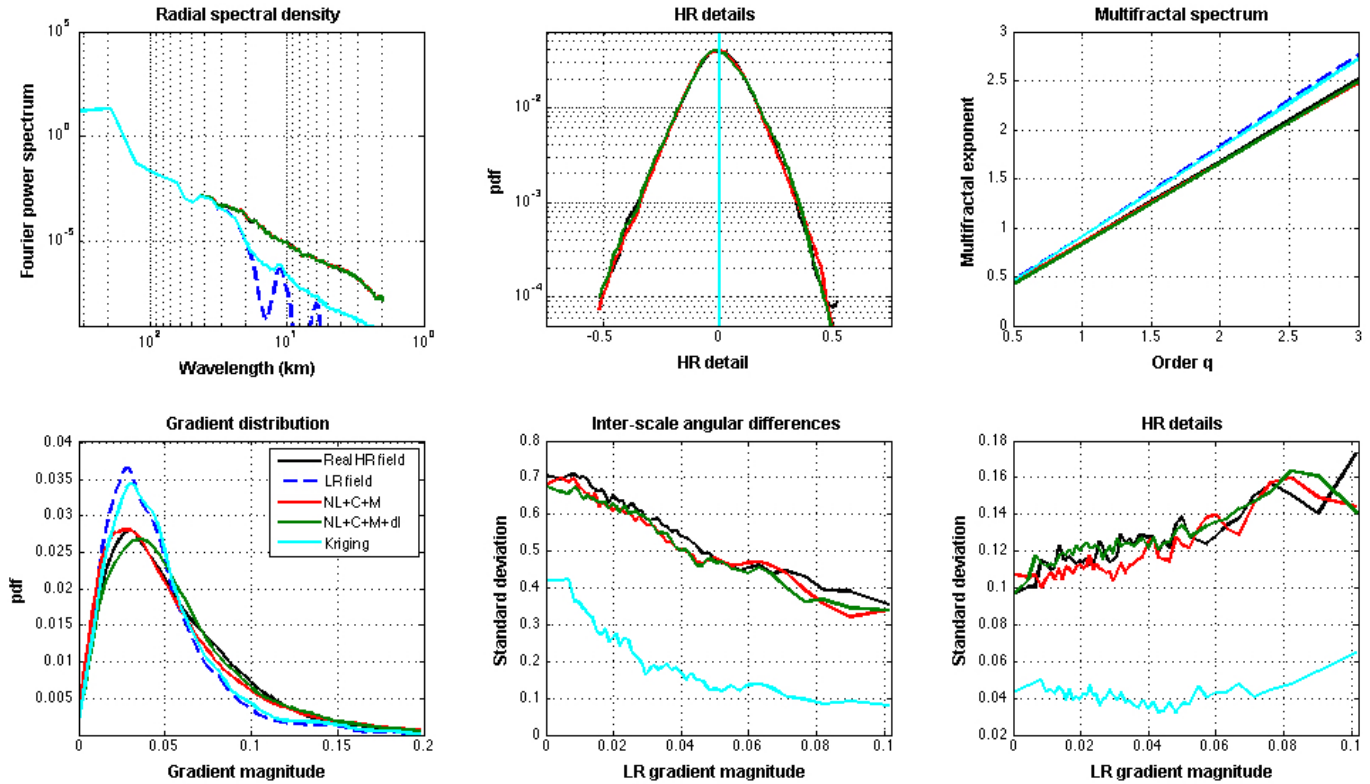

Fig. 5. Quantitative comparison of the interpolation results reported in Fig.4: we consider the same statistical features as in Fig.2.

the two preceding days. Other parameters are set similarly to the experiments on ODYSSEA SST data (Fig.).

We report in Fig.6 the interpolation results issued from the proposed model with a non-local projection applied to detail field $\delta \mathcal{P}_{W}^{L R}\left(X^{(2)}\right)$. Though the AMSR-E field involve few missing data, we benefit from the greater resolution of the SEVIRI field close to the shore to interpolate the AMSR-E field in these areas. This is particularly 

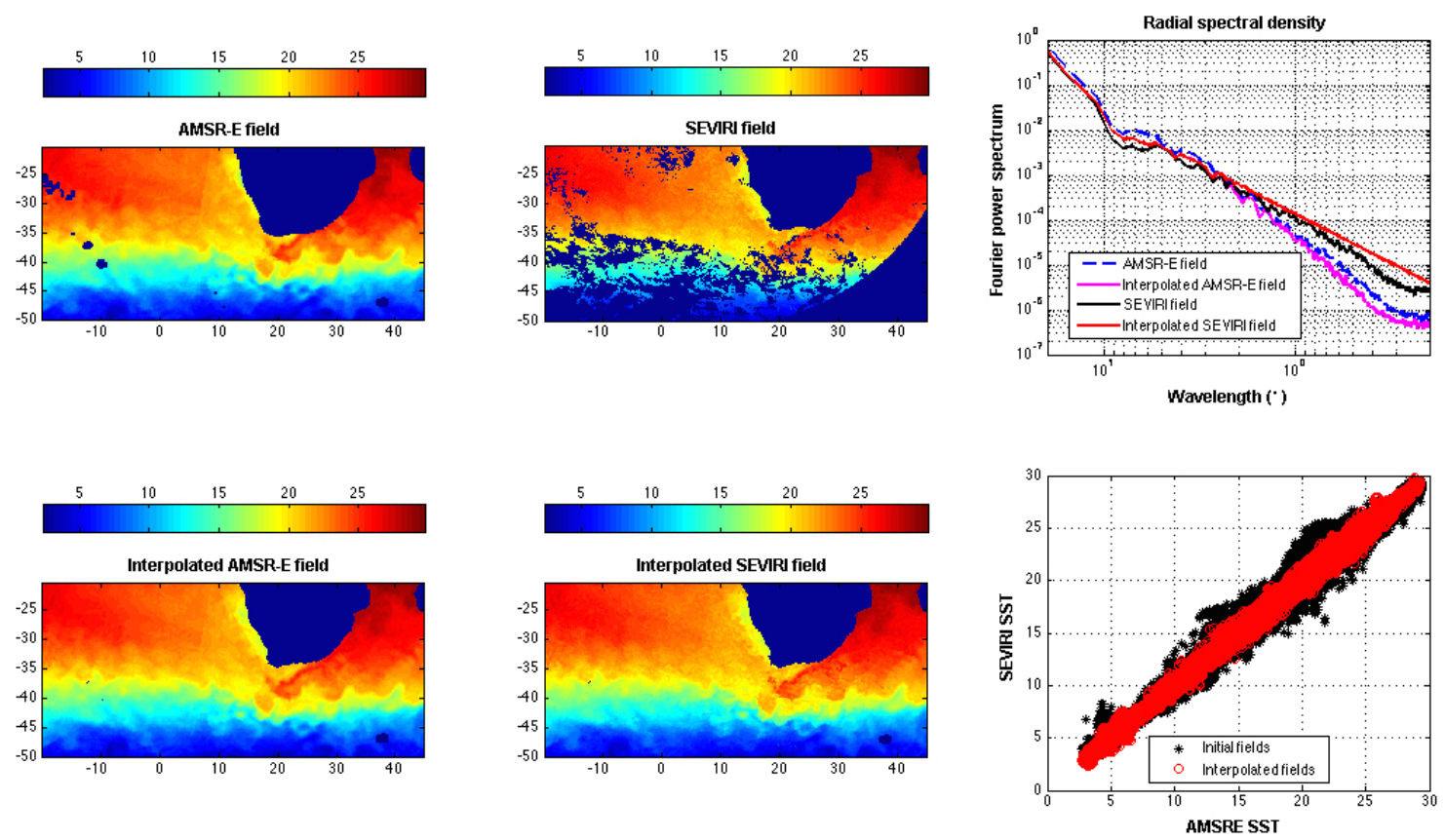

Fig. 6. Reconstruction of a daily SST field from a joint analysis of SEVIRI and AMSRE data: first row, AMSR-E field with few missing data (left), SEVIRI field field (center), radial power spectral density of the different fields (right); second row, interpolated AMSR-E field (left), interpolated SEVIRI field (center), scatterplot of AMSR-E and SEVIRI data for the original (black, *) and interpolated (red, o) fields (right).

noticeable off South Africa for the Aghulas current, characterized by warm waters going southward along the eastern coast of South Africa (see also, the first row of Fig.7). It may also be outlined that the constraint of a common low-resolution component between the interpolated AMSR-E and SEVIRI fields result in more coherent scatterplots of the low- vs high-resolution data (Fig.6, bottom right). From the visual inspection of the zoom onto an area off South Africa, involving an important missing data rate for the SEVIRI field (Fig.7), the gradient fields clearly stress the greater relevance of the combination of non-local and statistical priors in the south-east of the considered area. Besides, it may be noted that the application of the non-local projection to field $X^{(2)}$ produces unexpected tiling effects. This is due to a very low number of cold patches in the reference dataset. By contrast, applying the non-local projection to field $\delta \mathcal{P}_{W}^{s^{L R}}\left(X^{(2)}\right)$ leads to much more satisfactory interpolation results, as we are provided with more representative patch datasets for the detail field and a conditioning by the low-resolution gradient. The gradient fields also clearly stress the difference between the information carried out by AMSR-E and SEVIRI fields in terms of presence and location of frontal structures, with a much coarser resolution of the AMSR-E fields.

We report a second example of joint AMSR-E/SEVIRI interpolations for a date at which AMSR-E field also involves a large missing data area off South Africa (Fig.8). Following the conclusions drawn from the reported experiments, we only depict the zoom on the region off South Africa and the interpolation results for the proposed model with a non-local projection applied to detail field $\delta \mathcal{P}_{W}^{s^{L R}}\left(X^{(2)}\right)$. This example shows that we can interpolate 

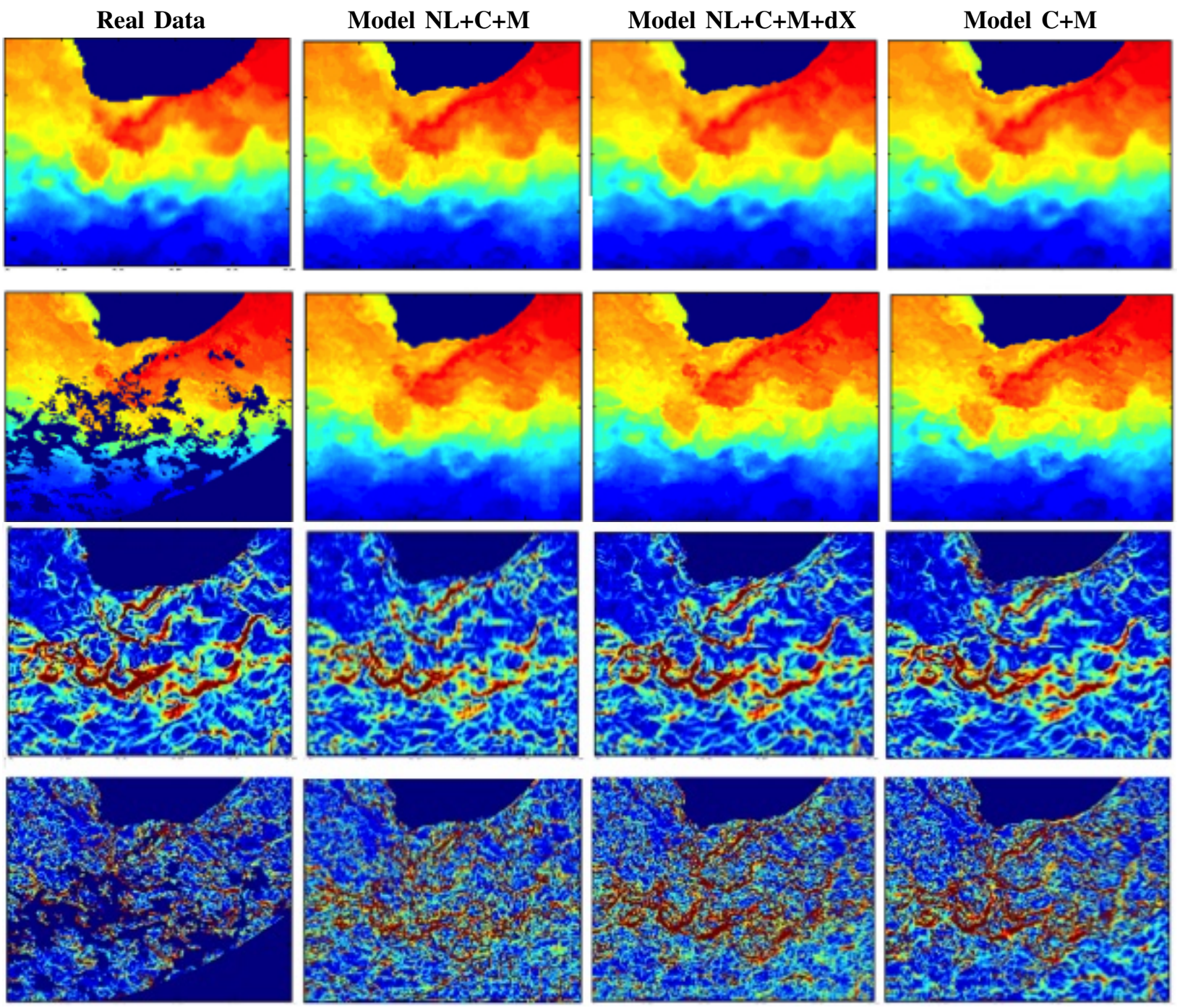

Fig. 7. Zoom of joint AMSR-E/SEVIRI interpolation results for the situation considered in Fig.6: first row, observed and interpolated AMSR-E fields; second row, observed and interpolated SEVIRI fields; third row, gradient of the AMSR-E fields, fourth row, gradient of the SEVIRI fields. For each column, we depict the observed data, the interpolation results using the proposed model with a non-local projection applied to field $X^{(2)}$ (Model $\left.\mathrm{NL}+\mathrm{C}+\mathrm{M}\right)$, the interpolation results using the proposed model with a non-local projection applied to detail field $\delta \mathcal{P}_{W}^{s^{L R}}\left(X^{(2)}\right)$ (Model $\mathrm{NL}+\mathrm{C}+\mathrm{M}+\mathrm{dX}$ ), the interpolation results using only the statistical priors (Model $\mathrm{C}+\mathrm{M}$ ).

visually-consistent fields both at AMSR-E and SEVIRI resolutions and jointly benefit from the two types of data to jointly improve the interpolation of the low-resolution and high-resolution fields.

\section{Discussion}

In this work, we have presented a novel model for the joint interpolation and blending of multi-source and multiresolution remote sensing images. As case-study, we have focused on sea surface temperature. The key contributions are two-fold: the development of non-local and exemplar-based approaches for this multimodal interpolation issues 

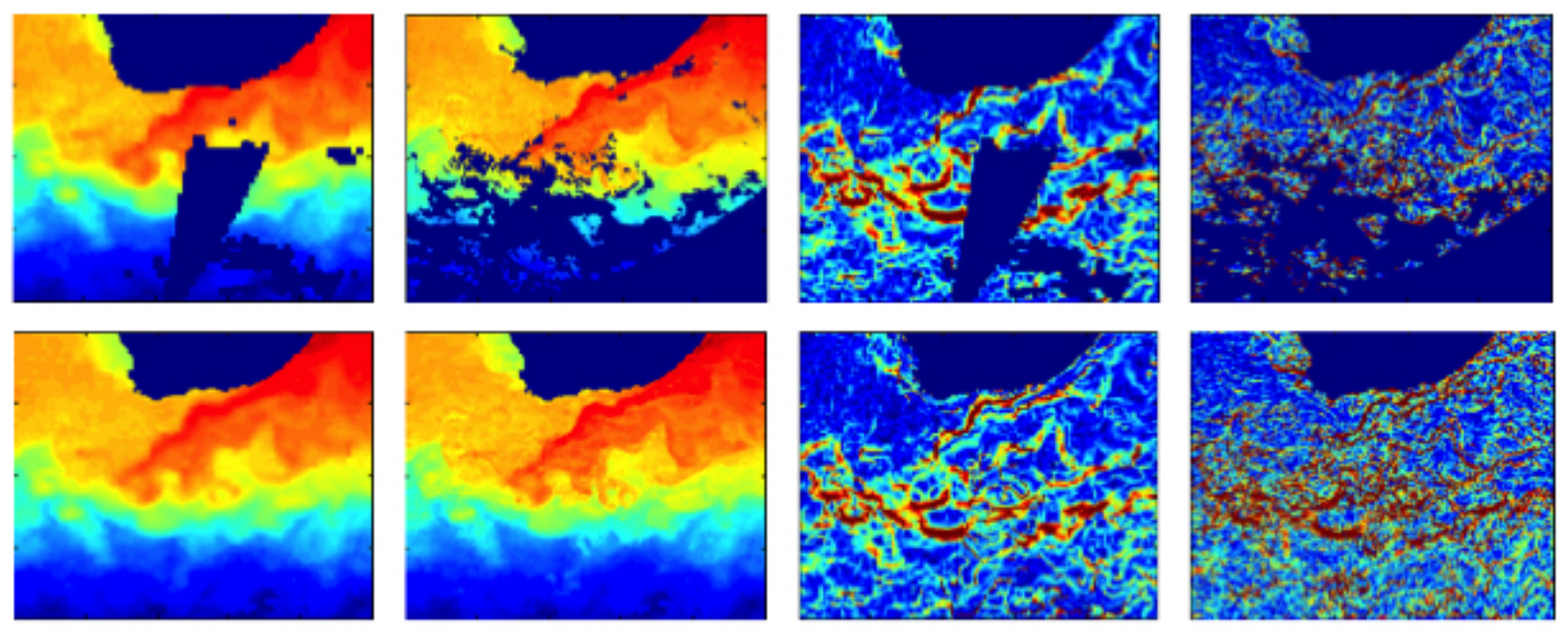

Fig. 8. Joint AMSR-E/SEVIRI interpolation results for a second situation involving large missing data areas for both sensors: first row, observed fields, second row, interpolated fields. For each row, we depict from left to right, the AMSR-E field, the SEVIRI field, the gradient of the AMSR-E field and the gradient of the SEVIRI field. We report a zoom on the same area as in Fig.7 off South Africa, which involves the largest missing data rate. The reported interpolation results are issued from the proposed model with a non-local projection applied to detail field $\delta \mathcal{P}_{W}^{L R}\left(X^{(2)}\right)$.

and the combination of these non-local priors to second-order statistical priors. The later is particularly motivated by the fact that sea surface fields are expected to depict specific fine-scale patterns associated with the advection of the geophysical tracers by turbulent flows. To our knowledge, this is the first work which addresses such combination between patch-based models and statistical priors for multi-resolution interpolation issues.

Reported results for both synthetic and real data demonstrate the relevance of this model compared to the sole use of non-local or statistical priors. Regarding the later, the prior on the spatial covariance of the high-resolution field is of greater importance than the prior on the marginal of the high-resolution details. We have also evaluated that the non-local projection applied to the detail field, rather than to the high-resolution field, offers a greater robustness with respect to possibly weakly represented high-resolution patches (in our case, patches corresponding to cold waters, Fig.7). The representativeness of the reference patch dataset is obviously a key issue for the effectiveness of the non-local prior. In this respect, we considered reference dataset with 5000 patches. Larger datasets may be considered at the expense of a greater computational cost. One may also explore spatialized version of the patch dataset, where patch similarities account not only for visual similarities but also for space and/or time similarities. Model parameters for the statistical priors can be fitted from the observed data and/or exploit priors on the considered turbulent dynamics for the spectral prior. The parameters of the non-local prior, especially the size of the patches, were here set empirically. Local patches between $9 \times 9$ and $13 \times 13$ were proven relevant. Larger patches typically result in border effects. The automatic learning and tuning of the patch similarity measure could be further explored as developed for other applications of non-local priors in computational imaging [32].

We reported a comparison of the proposed interpolation schemes to a kriging interpolation, classically used for 
ocean remote sensing data. As kriging-based model states the interpolation as a linear prediction of the missing data points from truly observed ones, it does not address the reconstruction of textural patterns, when the width of the missing data areas is above the spatial range of the considered covariance model (Fig.5). In operational applications, kriging and optimal interpolation involve a trade-off between the fidelity to the observed data and the interpolation of the missing data areas, with a view to delivering spatially-homogeneous fields. The proposed interpolation models develop a different strategy. We aimed at keeping all the relevant observations, while filling the missing data areas with geophysically-sound field patterns. Interestingly, we showed that the MSE statistics are comparable to a kriging interpolation for small missing data areas. Future work will further explore and evaluate this intrinsic difference with the classical geostatistical tools. In particular, we may consider interpolation ensembles from multiple random initializations. This would provide a mean to evaluate a mean field with persistent (deterministic) structures and some associated variabilities.

From a methodological point of view, our future work will also explore several extensions. Regarding the spectral prior, we only consider here radial (isotropic) covariance structure. The extension to parametric and non-parametric two-dimensional covariance structure could be explored. The same holds for other parametric models of the marginal distribution of the high-resolution detail. The extension to spatio-temporal fields will also be of key interest, including exemplar-based data assimilation issues [34]. Such extension might require manifold learning and kernel learning strategies to define a patch similarity measure adapted to the considered space-time processes [41].

Regarding the application to ocean remote sensing data, we focused here on SST datasets issued from two sensors. The extension of the proposed model to the joint interpolation of three or more data sources should be investigated. For instance, for SST data, operational ODYSSEA product typically aims at merging the daily data issued from at least 6 different sensors [2]. Such developments should involve particular methodological issues and could benefit from a classical coarse-to-fine implementation strategy to decompose this multi-sensor/multi-scale fusion as a sequence iteration of two-scale fusions. Applications to other sea surface geophysical fields (e.g., ocean colour, sea surface salinity) appear direct. Beyond the joint processing of multi-sensor data for same geophysical fields (here, SST), one could also explore the relevance of the proposed model for the joint analysis of different geophysical fields for which a mutual conditioning might be expected, but difficult to make explicit through a numerical model. The joint interpolation of SST and ocean colour data may provide a typical example. SST data might provide a mean to constrain the interpolation of ocean colour data, which often involve very large missing data rate in temperate areas.

\section{ACKNOWLEDGEMENTS}

This work was supported by ANR (Agence Nationale de la Recherche, grant ANR-13-MONU-0014). We are grateful to E. Autret, B. Chapron, J.F. Piolle from Ifremer/CERSAT for providing the SST data.

\section{REFERENCES}

[1] Alvera-Azcarate, A., Barth, A., Rixen, M., Beckers, J. M. Reconstruction of incomplete oceanographic data sets using empirical orthogonal functions: application to the Adriatic Sea surface temperature. Ocean Modelling, 9(4), 325-346, 2005. 
[2] Autret, E., Piolle, J. F. Product User Manual for ODYSSEA Level 3 and 4 global and regional products. MYO-PUM-SSTTAC-ODYSSEA, Ifremer/CERSAT, 2011. Available online at: http://projets.ifremer.fr/cersat/Data/Discovery/By-parameter/Sea-surfacetemperature/ODYSSEA-Global-SST-Analysis].

[3] Sirjacobs, D., Alvera-Azcarate, A., Barth, A., Lacroix, G., Park, Y., Nechad, B., Beckers, J. M. Cloud filling of ocean colour and sea surface temperature remote sensing products over the Southern North Sea by the Data Interpolating Empirical Orthogonal Functions methodology. J. Sea Res., 65(1):114-130, 2011.

[4] Ba, S., Autret, Chapron, B., Fablet, R. Statistical descriptors of ocean regimes from the geometric regularity of Sea Surface Temperature obser- vations. IEEE Geosc. Rem. Sens. Lett., 9(5):851-854, 2012.

[5] Buades, A., Coll, B., Morel, J.M. A non-local algorithm for image denoising. Proc. IEEE Conf. on Comp. Vis. Patt. Recog.. CVPR'2005, 2005.

[6] Bernard, D.,Boffetta, G., Celani, A., Falkovich, G. Conformal invariance in two-dimensional turbulence. Nature Physics, 2(2): 124-128, 2006.

[7] Byung Tae Oh, Jay Kuo, C.-C. Super-resolution texture synthesis using stochastic PAR/NL model. J Vis. Comp. Repr., 23(7):995-1007, 2012.

[8] Caselles, V., Morel, J.M., Sbert, C. An axiomatic approach to image interpolation.IEEE Trans. on Im. Proc., 7(3):376-386, 1998.

[9] Chainais, P. Konig, E., Delouille, V., Hochedez, J.-F. Virtual super resolution of textured images using multifractal stochastic processes. $J$. Math. Im. Vis., 39(1):28-44, 2011.

[10] Criminisi, A., Perez, P., Toyama, K. Region filling and object removal by exemplar-based image inpainting. IEEE Trans. on Image Processing, 13(9):1200-1212, 2004.

[11] Deledalle, C.-A., Denis, L., Tupin, F. NL-InSAR: Nonlocal interferogram estimation. IEEE Trans. on Geoscience and Remote Sensing, 49(4):1441-1452.

[12] Efros, A.A., Freeman, W.T. Image Quilting for Texture Synthesis and Transfer. Proc. ACM SIGGRAPH, 2001.

[13] Fablet, R., Boussidi, B., Autret, E., Chapron B. Random walk models for geometry-driven image super-resolution. Proc. 38th Int. Conf. on Acoustics, Speech and Signal Processing, ICASSP'2013, 2013.

[14] Freeman, W.T., Liu, C. Markov Random Fields for Super-resolution and Texture Synthesis. In A. Blake, P. Kohli, and C. Rother, eds., Advances in Markov Random Fields for Vision and Image Processing, Chapter 10. MIT Press, 2011.

[15] Galerne, B., Gousseau, Y., Morel., J.M. Random phase textures: Theory and synthesis. IEEE Trans. on Image Processing, 2011.

[16] Glasner, D., Bagon, S., Irani, M. Super-resolution from a single image.Proc. IEEE Int. Conf. on Computer Vision, ICCV'2009, 2009.

[17] He, H., Siu, W. C. Single image super-resolution using Gaussian process regression. Proc. IEEE Conf. on Comp. Vis. Patt. Rec., CVPR'2011, 449-456.

[18] Heeger, D.J., Bergen, J.R. Pyramid Based Texture Analysis/Synthesis. Proc. ACM SIGGRAPH, 229-238, 1995.

[19] Kolmogorov, A. N. (1941). The local structure of turbulence in in- compressible viscous fluid for very large reynolds numbers. Dokl. Akad. Nauk SSSR, 30:299-303, 1941.

[20] Lapeyre, G. and Klein, P. Dynamics of the upper oceanic layers in terms of surface quasigeostrophy theory. J. Phys. Oceanogr., 36(2):165176, 2006.

[21] Le Traon, P., Klein, P., Hua, B., and Dibarboure, G. Do al- timeter wavenumber spectra agree with the interior or surface quasigeostrophic theory? J. Phys. Oceanogr., 38(5):1137-1142, 2008.

[22] Le Meur, O., Ebdelli, M., Guillemot, C. Hierarchical Super-Resolution-Based Inpainting. IEEE Trans. on Image Processing, 22(10):37793790, 2013.

[23] Lguensat, R., Tandeo, P., Fablet, R., Garello, R. Spatio-temporal interpolation of Sea Surface Temperature using high resolution remote sensing data.Proc. IEEE OCEANS'2014, St. John's, Canada, sept. 2014.

[24] Lorenzi, L., Melgani, F. Mercier, G. Inpainting strategies for reconstruction of missing data in VHR images. IEEE Geoscience and Remote Sensing Lett., 8 (5):914-918, 2011.

[25] McCaffrey, K., Fox-Kemper, B., Forget, G. Estimates of ocean macro-turbulence: structure function and spectral slope from Argo profiling floats. J. Phys. Ocean., 2015.

[26] Mallat, S. A Wavelet Tour of Signal Processing. Acamdeic Press, 2008. 
[27] Nieves, V., Llebot, C., Turiel, A., Sole, J., Garcia-Ladona, E., Estrada, M., Blasco, D. Common turbulent signature in sea surface temperature and chlorophyll maps. Geophysical Res. Lett., 34(23), 2007.

[28] Stark, J. D., Donlon, C. J., Martin, M. J., McCulloch, M. E. OSTIA: An operational, high resolution, real time, global sea surface temperature analysis system. Proc. IEEE Oceans, 2007.

[29] Peyre, G., Bougleux, S., Cohen, L. Non-local Regularization of Inverse Problems. Proc. Eur. Conference on Comp. Vis., ECCV’2008, 57-68, 2008.

[30] Qi, P., Cao, L. The assimilation of Jason-2 significant wave height data in the north Indian ocean using the ensemble optimal interpolation, IEEE TGRS, 54(1), 2016.

[31] Romano, Y., Protter, M., Elad, M. Single Image Interpolation Via Adaptive Nonlocal Sparsity-Based Modeling. IEEE Trans. on Image Processing, 23(7):3085-3098, 2014.

[32] Rousseau, F. A non-local approach for image super-resolution using intermodality priors. Medical Image Analysis, 14(4):594-605, 2010.

[33] P. Tandeo, P. Ailliot and E. Autret, Linear Gaussian State-Space Model with Irregular Sampling: Application to Sea Surface Temperature, Stochastic Environmental Research and Risk Assessment, 25(6), 793-804, 2011.

[34] Tandeo, P., Ailliot, P., Ruiz, J., Hannart, A., Chapron, B., Cuzol, A., Monbet, V., Easton, R., Fablet, R.. Combining analog method and ensemble data assimilation: application to the Lorenz-63 chaotic system. in Machine Learning and Data Mining Approaches to Climate Science, Springer, 2015.

[35] Tartavel, G., Gousseau, Y., Peyré, G. Variational Texture Synthesis with Sparsity and Spectrum Constraints JMIV, 52(1): 124-144, 2015.

[36] Wang, D., Lang, S. Improving LAI mapping by integrating MODIS and CYCLOPES LAI products using optimal interpolation, IEEE JTARS, 7(2), 2014.

[37] Xu, Y., L.-L. F. Global variability of the wavenumber spectrum of oceanic mesoscale turbulence. J. Phys. Oceanogr., 41 :802-809, 2011.

[38] Yi, L., Takahashi, K., Sato, M. A fast iterative interpolation method in f-k domain for 3-D irregularly sampled GPR data, IEEE JSTARS, 2015. DOI: $10.1109 /$ JSTARS.2015.2489260.

[39] Yu, W., Nan, Z., Wang, Z., Chen, H., Wu, T., Zhao, L. An effective interpolation method for MODIS land surface temperature on the Qinghai-Tibet Plateau, IEEE JSTARS, 2015. DOI: 10.1109/JSTARS.2015.2464094.

[40] Zhang, D. Tang, R., Tang, B.H., Wu, H. A simple method for soil moisture determination from LST-VI feature space using nonlinear interpolation based on thermal infrared remotely sensed data. IEEE JSTARS, vol. 8, no. 2, 2015.

[41] Zhao, Z., Giannakis, D. Analog forecasting with dynamics-adapted kernels. arXiv preprint arXiv:1412.3831, 2014

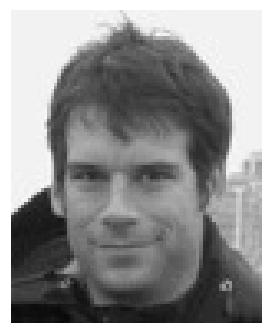

Ronan Fablet graduated from the Ecole Nationale Supérieure de l'Aéronautique et de l'Espace (SUPAERO), Toulouse, France, in 1997. He received the Ph.D. degree in signal processing and telecommunications from the University of Rennes, Rennes, France, in 2001.

In 2002, he was an Institut National de Recherche en Informatique et Automatique Postdoctoral Fellow with Brown University, Providence, RI, USA. From 2003 to 2007, he held a full-time research position at IFREMER Brest in the field of signal and image processing applied to fisheries science. In 2008, he joined the Signal and Communications Department, Telecom Bretagne, as an Associate Professor, and has been holding a Professor position since 2012. In 2011, he was a Visiting Researcher at Institut de Recherche pour le Développement/Instituto del Mar del Peru, Peru (Peruvian Sea Research Institute). His main research interests include statistical methods for signal processing and computer vision and applications to ocean remote sensing. 


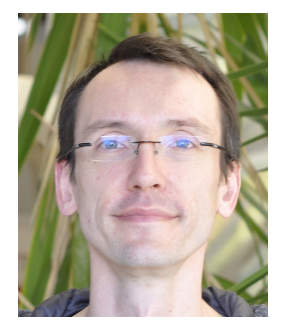

François Rousseau is a Professor at Télécom Bretagne / Institut Mines Télécom since 2015. He obtained his PhD in 2003 (University of Rennes) and his Habilitation in 2014 (University of Strasbourg). He worked at the University of California San Francisco in 2004?2005, and then at ICube lab (Université de Strasbourg) as a CNRS research scientist between 2006-2015. His research focuses on image analysis, and more specifically on image reconstruction, segmentation, temporal data and computational anatomy.

\section{SUPPLEMENTARY MATERIAL}

We provide in this annex the detailed iterative algorithm used to solve for constrained minimization (15)). More precisely, we describe in the subsequent:

- Algorithm (1): the algorithm of function projection_Interp_LR which solves for the projection onto the interpolation and low-resolution constraints (first two constraints in (16));

- Algorithm (2): the algorithm of function projection_PSD_PDF used to solve for the projection onto the spectral and marginal constraints (last two constraints in (16));

- Algorithm (3): the general iterative algorithm of the proposed approach for the joint interpolation and blending of two multi-sensor fields

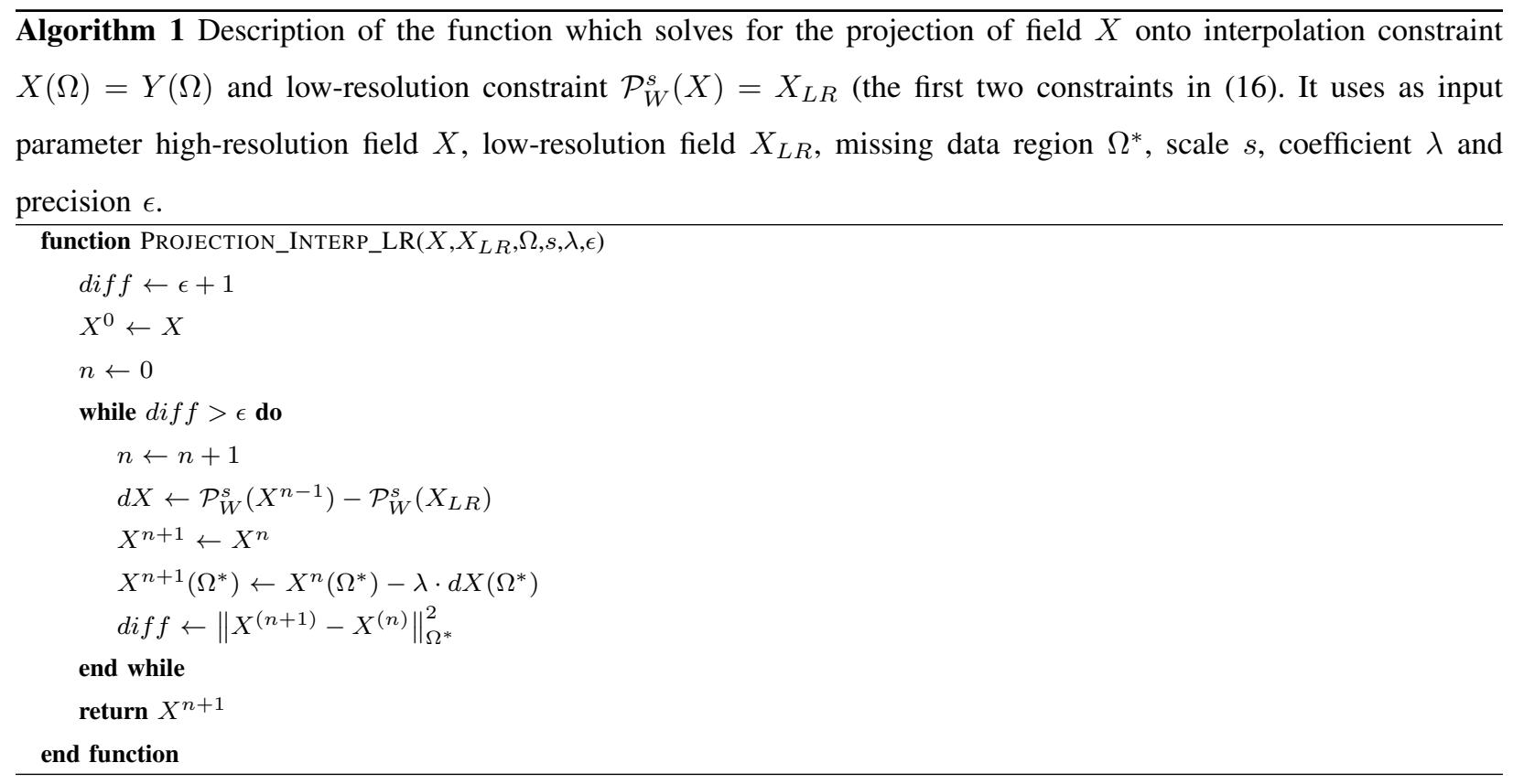




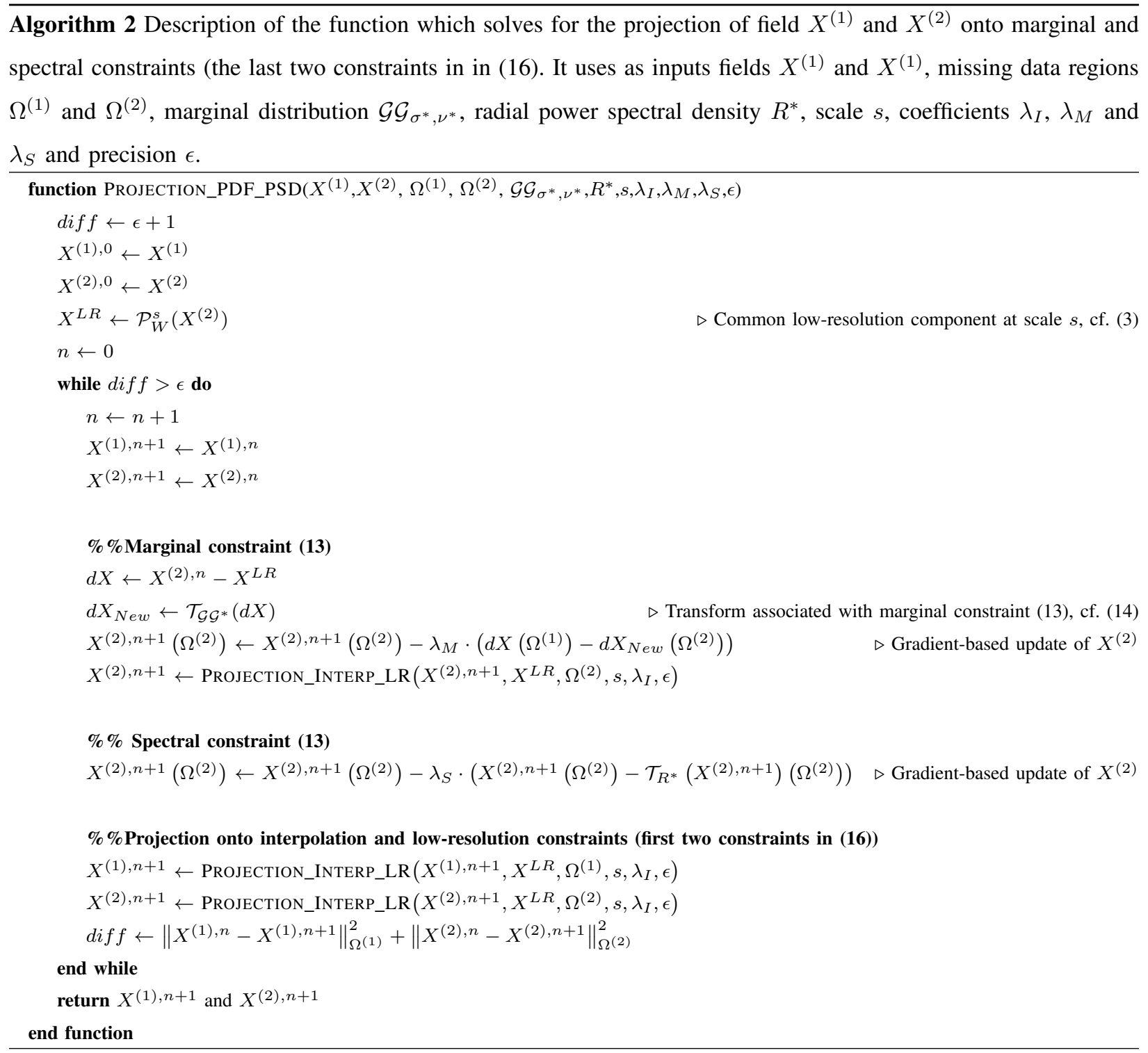




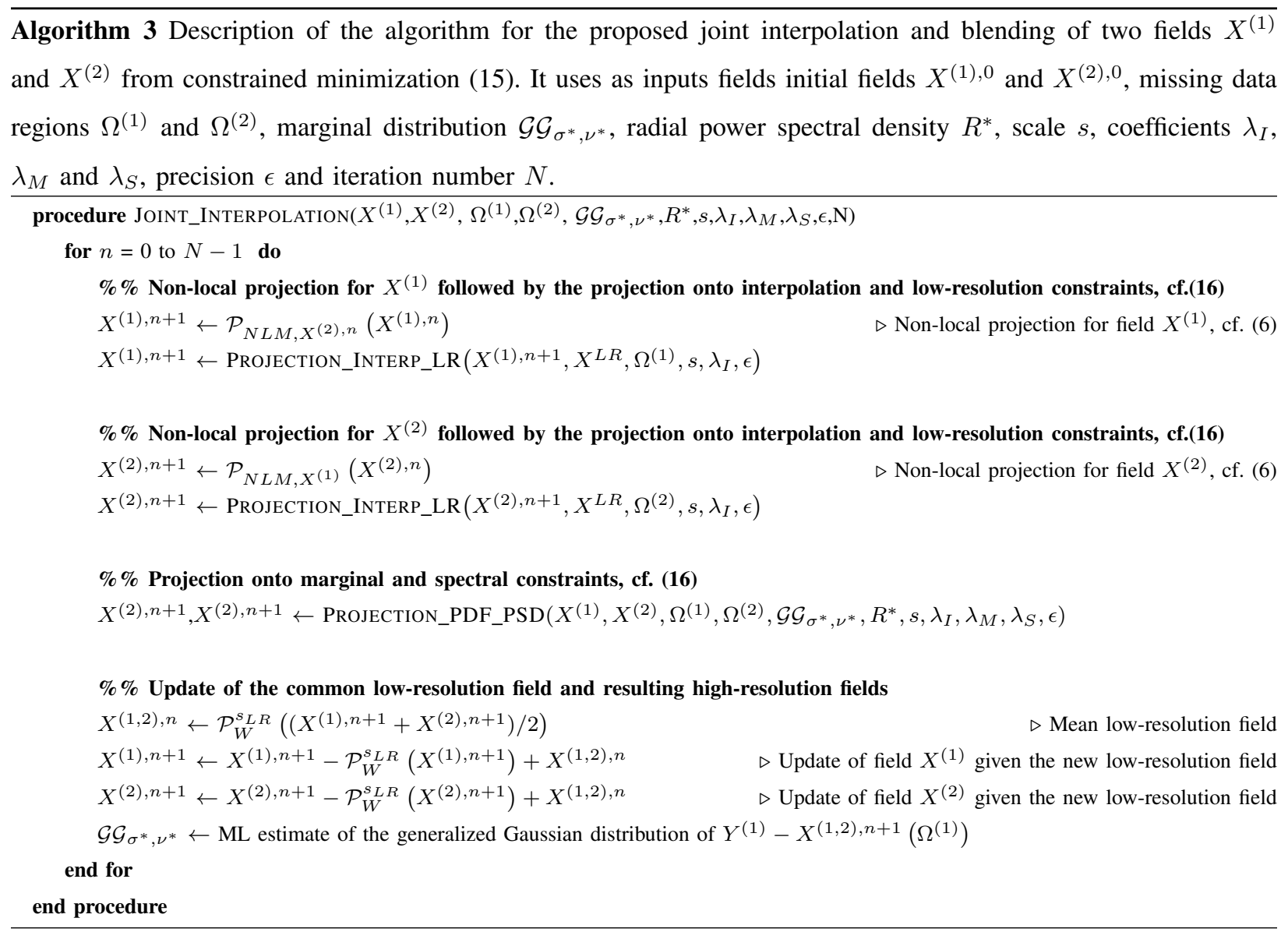

